\title{
A quality improvement initiative to increase the use of patients' own drugs through the implementation of a 'Green Bag' scheme at a central London hospital
}

\author{
Authors: Marios Erotocritou, ${ }^{\mathrm{A}}$ George Choa, ${ }^{\mathrm{A}}$ Oliver I Clark, ${ }^{\mathrm{A}}$ Fatema Mamdani, ${ }^{\mathrm{B}}$ Lyndsey Nicoll,, ${ }^{\mathrm{B}}$ Saika Walji, \\ Tan Yi Xin, ${ }^{B}$ Gemma Wareing ${ }^{C}$ and Yogini Jani ${ }^{C}$
}

Aims

Aims were:

$>$ primarily to increase the usage of patients' own drugs (PODs) throughout elective surgery patients' hospital stay via the use of the Green Bag scheme (which has demonstrated positive results in other hospitals)

$>$ improvement of medication reconciliation

$>$ reduction in administrative delays

$>$ reduction in drug costs and waste

$>$ improving patients' experience

$>$ provide patients with a physical reminder and means of bringing all their PODs when admitted for surgery

$>$ create an easily identifiable source of their medicines for clinical staff.

\section{Methods}

An audit was conducted at a central London hospital. Baseline data were collected in a retrospective manner using a pro forma. Baseline data about POD usage, prior to implementation of the Green Bag scheme, were collected from 305 patients undergoing elective surgery between August and October 2016. The scheme was introduced to patients attending the preoperative assessment clinic (POAC) from December 2016 onwards. Post-implementation data were collected by surgical reception staff. Subsequently, we introduced a second plan, do, study, act (PDSA) cycle with the addition of information leaflets regarding the scheme and its potential benefits; addressing both patients and staff. As a third PDSA cycle, surgical reception pharmacists were recruited to improve the accuracy and precision of data collection on POD utilisation. In total, post-implementation data were collected for 316 patients over three PDSA cycles. With exclusion criteria applied, data were analysed and run charts were plotted against the baseline data.

Authors: ${ }^{A}$ Medical School, University College London, London,

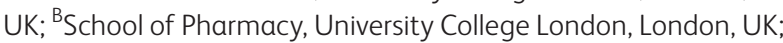
${ }^{C}$ Centre for Medicines Optimisation Research \& Education, UCLH NHS Foundation Trust, London, UK

\section{Results}

We have noted a general trend of improvement in the data throughout the study. We noted a higher proportion of patients bringing their PODs when given a green bag $(77.3 \%)$ in comparison with patients who were not given one $(60.8 \%)$, in line with our hypothesis. Similarly, as expected, less doses of PODs were missed with fewer additional drugs dispensed during a patient's stay and on discharge. Therefore, the scheme reduced discharge times and length of stay.

\section{Conclusion}

The Green Bag scheme proved to be effective at achieving our study aims. All the above findings suggest potential major cost savings to the hospital through decreased medication errors, decreased hospital-dispensed medications, and decreased length of stay in hospital.

A major limitation of this project was the targeted hospital population of elective surgery. Collecting data in this population was difficult, as $70 \%$ of available patient data was lost due to our exclusion criteria.

\section{Conflict of interest statement}

None declared. 